\title{
A note on the Weibel instability and thermal fluctuations
}

\author{
R. A. Treumann ${ }^{1,2}$ and W. Baumjohann ${ }^{3}$ \\ ${ }^{1}$ Department of Geophysics and Environmental Sciences, Munich University, Munich, Germany \\ ${ }^{2}$ Department of Physics and Astronomy, Dartmouth College, Hanover NH 03755, USA \\ ${ }^{3}$ Space Research Institute, Austrian Academy of Sciences, Graz, Austria \\ Correspondence to: R. A. Treumann (rudolf.treumann@geophysik.uni-muenchen.de)
}

Received: 24 January 2012 - Accepted: 24 February 2012 - Published: 28 February 2012

\begin{abstract}
The thermal fluctuation level of the Weibel instability is recalculated. It is shown that the divergence of the fluctuations at long wavelengths, i.e. the Weibel infrared catastrophe, never occurs. At large wavelengths the thermal fluctuation level is terminated by the presence of even the smallest available stable thermal anisotropy. Weibel fields penetrate only one skin depth into the plasma. When excited inside, they cause layers of antiparallel fields of skin depth width and vortices which may be subject to reconnection.
\end{abstract}

Keywords. Space plasma physics (Waves and instabilities)

\section{Introduction}

The Weibel thermal (Weibel, 1959) and Weibel filamentation (Fried, 1959) instabilities, two closely related versions of the purely electromagnetic zero frequency instability of a non-magnetised plasma, have been proposed (see, e.g. Yoon and Davidson, 1987) as fundamental for the generation of quasi-stationary magnetic fields (Medvedev and Loeb, 1999) in high temperature or high-speed (cf., e.g. Achterberg and Wiersma, 2007) plasmas, respectively. The mechanism of this instability has been elucidated (Fried, 1959) as the spontaneous microscopic electron (or ion) currents flowing in a plasma which, under unstable conditions, become amplified and cause a non-vanishing magnetic field to grow in the plasma.

As usual, the field grows up to a maximum amplitude and stabilises by some mechanism like exhaust of the available free energy, in the case of the thermal Weibel mode quasilinear depletion of the temperature anisotropy (recent particularly complete discussions are given in Pokhotelov et al., 2010; Pokhotelov and Amariutei, 2011), in the case of the Weibel filamentation mode by self-magnetisation of the initially non-magnetised streaming particles (cf., e.g. Achter- berg and Wiersma, 2007). The generated fields are (quasi)stationary on temporal scales longer than growth and stabilisation times. Nonlinear effects set on when the waves reach large amplitudes after amplification. At this stage, quasilinear modification of the distribution function limits further growth (Pokhotelov and Amariutei, 2011) and wave-wave interaction causes further nonlinear evolution (Pokhotelov et al., 2010) which distributes particle energy into other modes, further limiting the growth of magnetic fields.

Thus, under non-dynamo conditions, the Weibel mechanism is an alternative to the celebrated turbulent magnetic dynamo mechanism, the latter being believed to be responsible for the generation of magnetic fields in planets (Christensen et al., 2010; Hulot et al., 2011), stars (e.g. Jones et al., 2010), galaxies and on the large scale in the universe. The Weibel instability may grow in the universe from thermal fluctuations at early times (Widrow et al., 2011) either in the chromodynamic state or later in the plasma state, and also after recombination during structure formation (Ryu et al., 2011). It is believed to dominate all other mechanisms in magnetic field generation in relativistic shocks (cf., e.g. Bykov and Treumann, 2011). More recently, it has been suggested to play a decisive role in the ignition of magnetic reconnection in collisionless current sheets (Baumjohann et al., 2010; Treumann et al., 2010).

These facts, in particular the astrophysical application to magnetic field generation in shocks, let the Weibel instability attract considerable interest. Nevertheless, several open questions remain in dealing with it. These refer to the instability itself, independent of its free energy source, i.e. whether thermal or flow driven, and to its thermal level. In the following we very briefly clarify two of these: the nature of the Weibel mode, and avoiding the infrared catastrophe of Weibel thermal fluctuations. 


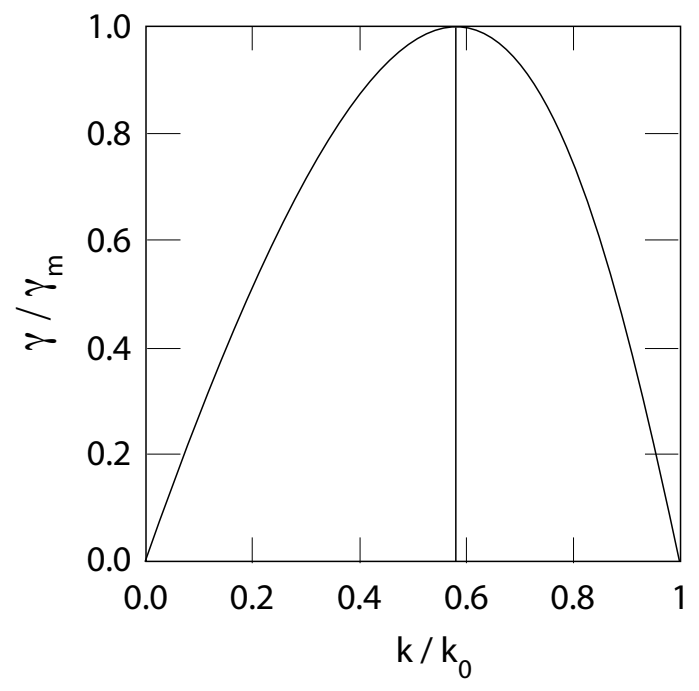

Fig. 1. Dependence of the normalised (to maximum growth $\gamma_{\mathrm{m}}$ ) Weibel growth rate $\gamma$ on wavenumber. The domain of growing wave numbers is limited by the skin-dpeth wave number $k_{\mathrm{e}}=\omega_{\mathrm{e}} / c$.

\section{Weibel mode}

In which mode does the Weibel instability propagate? This question is non-trivial because, in unmagnetised plasma of density $N$, electromagnetic wave propagation becomes possible only at frequencies above the plasma frequency $\omega_{\mathrm{e}}=$ $\sqrt{e^{2} N / \epsilon_{0} m_{\mathrm{e}}}$, a condition which immediately follows from the unmagnetised plasma wave dispersion relation $\omega^{2}=$ $k^{2} c^{2}+\omega_{\mathrm{e}}^{2}$, with $k$ wavenumber, and $c$ velocity of light. Zero frequency modes cannot propagate in this case. As a byproduct this implies that no magnetic fields can be imposed into the collisionless plasma. They must have been present from the very beginning, i.e. they must have been born with the plasma. Otherwise magnetic fields can enter only a distance of the order of the electron skin (inertial) depth, $\lambda_{\mathrm{e}}=c / \omega_{\mathrm{e}}$ in the collisionless case. Such waves in the plasma are evanescent.

As is well known, the situation changes, when the plasma is already magnetic, containing a constant magnetic field $\boldsymbol{B}$, in which case Alfvén, whistler, and magnetosonic waves can propagate down to extremely low frequency. Hence, what is the reason for the Weibel instability to survive and cause real magnetic fields in plasma?

In order to understand this point, we briefly repeat the thermal Weibel theory giving it a simple representation. Thermal Weibel instability requires that the plasma temperature is for some unspecified reason - anisotropic, allowing to define two temperatures $T_{\|}, T_{\perp}$ in orthogonal directions. For instance, if the plasma streams with velocity $\boldsymbol{V}$, then the plasma pressure might become anisotropic,

$\mathrm{P}_{\mathrm{e}}=n k_{B}\left[T_{\mathrm{e} \perp} \mathrm{I}+\left(T_{\mathrm{e} \|}-T_{\mathrm{e} \perp}\right) \boldsymbol{V} \boldsymbol{V} / V^{2}\right]$ (with $\mathrm{I} \equiv \delta_{i j},(i, j)=1,2,3$ the unit tensor) thus defining anisotropic temperatures parallel and perpendicular to the flow, yielding a bi-Maxwellian equilibrium distribution. The free energy stored in the anisotropy is available for driving instability. The linear dispersion relation (see, e.g. Baumjohann and Treumann, 2012), from which instability can be inferred, becomes

$D(\omega, \mathbf{k})=\left(n^{2}-\epsilon_{\perp}\right)^{2} \epsilon_{\|}=0, \quad n^{2}=k^{2} c^{2} / \omega^{2}$

where $\epsilon_{\perp}(\omega, \boldsymbol{k}), \epsilon_{\|}(\omega, \boldsymbol{k})$ are the transverse and longitudinal response functions. The longitudinal function is of no interest as it does not yield electromagnetic waves. The transverse response function is, using the bi-Maxwellian, given by

$\epsilon_{\perp}(\omega, \boldsymbol{k})=1+\frac{\omega_{\mathrm{e}}^{2}}{\omega^{2}}\{1-(A+1)[1+\zeta Z(\zeta)]\}-\frac{\omega_{\mathrm{i}}^{2}}{\omega^{2}}$

Here $Z(\zeta)$ is the plasma dispersion function of argument $\zeta=$ $\omega / k_{\perp} v_{\mathrm{e} \perp}$, and $A=T_{\mathrm{e} \|} / T_{\mathrm{e} \perp}-1>0$ the thermal anisotropy, $v_{\mathrm{e}}^{2}=2 T_{\mathrm{e}} / m_{\mathrm{e}}$ the thermal velocity, and $\boldsymbol{k}=(k \sin \theta, 0, k \cos \theta)$ is the wave vector having only two components. At very low frequencies $\omega \approx 0$ the unstable solution obtained by putting $\omega(\boldsymbol{k})=i \gamma(\boldsymbol{k})$ purely imaginary, yields for the Weibel growth rate

$\gamma_{\mathrm{WI}}\left(k_{\perp}\right)=\sqrt{\frac{2}{\pi}} \frac{k_{\perp} v_{\mathrm{e} \perp}}{k_{0} \lambda_{\mathrm{e}}}\left(1-\frac{k_{\perp}^{2}}{k_{0}^{2}}\right)(A+1)\left(k_{0} \lambda_{e}\right)^{3}$

With $A>0$, the wave will grow as long as $k_{\perp}<k_{0}$, where $k_{0} \lambda_{\mathrm{e}}=\sqrt{A}$ limits the unstable range in wavenumber. Maximum growth is obtained at $k_{\mathrm{m}}=k_{0} / \sqrt{3}$, close to the instability cut-off, This is shown in Fig. 1 with maximum growth rate

$\gamma_{\mathrm{m}}\left(k_{\mathrm{m}}\right)=\omega_{\mathrm{e}} \sqrt{\frac{8 A^{3}}{27 \pi}}\left(\frac{v_{\mathrm{e} \perp}}{c}\right)(A+1) \ll \omega_{\mathrm{e}}$

thus justifying the initial assumption of small frequency, but the unstable waves being of wavelength somewhat larger than the plasma skin depth.

We note in passing that the Weibel filamentation growth rate for two equal density counter streaming electron flows of speed $V_{\mathrm{b}}$ becomes

$\gamma_{\mathrm{FI}}=k_{\mathrm{be}} V_{\mathrm{b}}\left\{1+\frac{\omega_{\mathrm{be}}^{2}}{k^{2} c^{2}}\left[1+\frac{2 N_{\mathrm{e}}}{N_{\mathrm{b}}}\left(1+\frac{m_{\mathrm{e}}}{m_{\mathrm{i}}}\right)^{-1}\right]\right\}^{-\frac{1}{2}}$

where the index "b" refers to the two counter-streaming plasmas. In the long-wavelength range, if the background density is large, $N_{\mathrm{e}}>N_{\mathrm{b}} / 2$, this expression simplifies to

$\frac{\gamma_{\mathrm{FI}}}{k_{\mathrm{be}} V_{\mathrm{b}}} \lesssim k \lambda_{\mathrm{eb}} \sqrt{\frac{N_{\mathrm{b}}}{2 N_{\mathrm{e}}}}\left(1-\frac{N_{\mathrm{b}}}{4 N_{\mathrm{e}}}\right) \equiv k \lambda_{\mathrm{b}}$

where $k_{\mathrm{be}}=\omega_{\mathrm{be}} / c=\lambda_{\mathrm{be}}^{-1}$. This growth rate is small, unless $V_{\mathrm{b}}$ is large. The plot of the growth rate is given in Fig. 2. It 


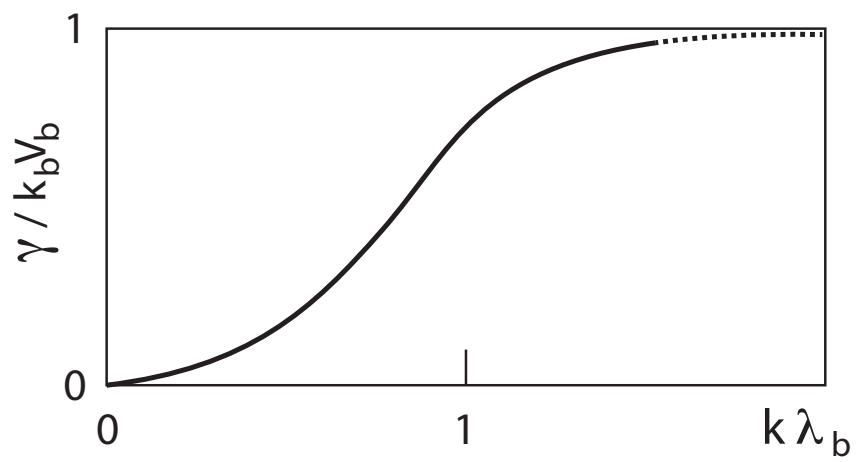

Fig. 2. The growth rate of the Weibel filamentation instability as function of wavenumber $k \lambda_{\mathrm{b}} \equiv k \lambda_{\mathrm{eb}} \sqrt{N_{\mathrm{b}} / 2 N_{\mathrm{e}}}$.

shows, in contrast to the thermal Weibel mode, that the linear growth rate is not restricted in the first place but formally extends to the limit $\gamma_{\mathrm{FI}} / k_{\mathrm{be}} V_{\mathrm{b}}=k \lambda_{\mathrm{b}}$ for $k \rightarrow \infty$. Of course, a natural upper bound of $k<k_{\mathrm{D}}$ is provided by the Debye wave number $k_{\mathrm{D}}$.

From this one concludes that, in a strictly non-magnetised plasma, the Weibel mode, whether thermal or flow driven, is not a bulk plasma mode. Under unstable conditions when it is excited in the plasma at some location $x$, its magnetic field will be felt only in an interval $\left[x-\lambda_{\mathrm{e}}, x+\lambda_{\mathrm{e}}\right]$ around $x$, producing magnetic fields in a region of the size of $\sim 2 \lambda_{\mathrm{e}}$.

Therefore, in an extended plasma containing temperature anisotropies, the Weibel mode will necessarily cause filamentary magnetic field structures, even when excited by thermal anisotropies. These structures, because of the zero divergence requirement are located adjacently carrying Weibel magnetic fields of opposite direction on transverse scales of the electron skin depth. Such structures resemble mutually adjacent current layers, as have been observationally inferred, for instance in the magnetosheath (Retinò et al., 2007). They may become unstable against reconnection, form magnetic islands and dissipate part of the free energy by transforming it into plasma heat, depletion of temperature anisotropy or kinetic flow energy, and related effects.

\section{Thermal fluctuation effects}

The Weibel instability is not really of zero-frequency. An estimate of the small though finite real frequency $\omega_{\mathrm{r}}$ is obtained from the real part of the dispersion relation (formally assuming $\gamma \gg \omega_{\mathrm{r}}$ ) under the simplifying assumption that $\omega \ll \omega_{\mathrm{e}}$. This yields

$\omega_{\mathrm{r}} \approx k_{\perp} c\left(\frac{v_{\mathrm{e} \perp}}{c}\right) \sqrt{\frac{1}{2} \frac{A+m_{\mathrm{e}} / m_{\mathrm{i}}}{A+1}}$

Since $v_{\mathrm{e}} / c \ll 1$ this shows that the frequency is very small, the index of refraction is $n>1$, and the wave, if propagating at all, must propagate on one of the low frequency magne- tised plasma branches, Alfvén or whistler. The long wavelength electron whistler dispersion relation is

$\frac{k^{2} c^{2}}{\omega^{2}} \approx \frac{\omega_{\mathrm{e}}^{2}}{\omega \omega_{\mathrm{ce}}}$

with $\omega_{\mathrm{ce}}=e B / m_{\mathrm{e}}$ the electron cyclotron frequency. In order for the Weibel mode to propagate at such low frequency as a whistler (or ultimately electron Alfvén) wave, this requires that the plasma must be weakly magnetised initially and

$k_{\perp} V_{\mathrm{Ae}} / \omega_{\mathrm{e}} \approx \sqrt{A /(A+1)}$

determines the wave number, with $V_{\mathrm{Ae}}$ the electron Alfvén speed. This becomes possible once the Weibel instability has grown in the above spatial slot $\left[x-\lambda_{\mathrm{e}}, x+\lambda_{\mathrm{e}}\right]$.

However, initially, the plasma is not completely free of magnetic fields. Thermal fluctuations provide a weak spontaneous magnetic fluctuation background level. This has been known for long time (Landau and Lifshitz, 1959, 1960) for any thermal electrodynamically active medium. The explicit theory of thermal fluctuations in plasmas can be found in Sitenko (1967) and Akhiezer et al. (1975). Application to magnetic fluctuations has been given by Yoon (2007) and Treumann et al. (2010).

Being a thermal dielectric response property, thermal fluctuations can exist in regions, where real waves do not propagate. Otherwise, they provide a pool of initial rms wave amplitudes, $\bar{b}=\sqrt{\left\langle b^{2}\right\rangle}$, from which the instability chooses to excite a particular range of wave modes which are allowed to propagate. For the Weibel mode this property has already been exploited (Treumann et al., 2010).

On the other hand, the weak magnetic fluctuation level, $\bar{b}$, also provides an average finite weak background magnetic field which can be chosen by the unstable wave to propagate. Taking this fluctuation wave field, the background Alfvén velocity becomes $\left\langle V_{\mathrm{A}}\right\rangle=\bar{b} / \sqrt{\mu_{0} m_{\mathrm{e}} N_{\mathrm{e}}}$, which enters the above whistler dispersion relation and fixes the wave number of the unstable modes which then may, indeed, propagate.

Since the frequency becomes very low, implying that also $k_{\perp} \rightarrow 0$, when interested in the generation of quasi-stationary magnetic fields, the magnetic fluctuation spectrum must be calculated slightly more precisely than done in the above references. There the magnetic fluctuation level depended on wave number as $\left\langle b^{2}\right\rangle \propto\left(k_{\perp} \lambda_{\mathrm{e}}\right)^{-3}$, leading to an "infrared catastrophe" for vanishing $k_{\perp}$ and quasi-stationary fields.

Such a catastrophe cannot occur in nature. A more precise calculation should take into account two facts. First, the plasma also contains ions (which have been included in the above transverse response functions but have not yet been used). Second, calculation of thermal fluctuations in the thermodynamic equilibrium (or quasi-equilibrium) state for the stable thermal Weibel mode implies

$A=T_{\|} / T_{\perp}-1<0$ 
The transverse response function can be written as

$\epsilon_{\perp}=1+\frac{\omega_{\mathrm{e}}^{2}}{\omega^{2}}\left\{1-\frac{T_{\|}}{T_{\perp}}\left[1-\Phi(z)+i \sqrt{\pi} z \mathrm{e}^{-z^{2}}\right]\right\}-\frac{\omega_{i}^{2}}{\omega 2}$

where $z=\zeta / \sqrt{2}$, and $\Phi(z) \approx 2 z^{2}$ for $z \ll 1$. Then, in the very low frequency limit, $\omega \rightarrow 0$, one obtains the correct thermal spectral energy density of magnetic fluctuations in the anisotropic-pressure plasma as

$$
\left(b^{2}(k)\right)=\frac{\mu_{0}}{\omega_{\mathrm{e}}} \sqrt{\frac{\pi T_{\perp}}{m_{\mathrm{e}} c^{2}}} \frac{m_{\mathrm{e}} c^{2}(A+1)^{2} k \lambda_{\mathrm{e}}}{(A+2)\left[k^{2} \lambda_{\mathrm{e}}^{2}-A-m_{\mathrm{e}} / m_{\mathrm{i}}\right]^{2}}
$$

For $A>0$ we had $k^{2} \lambda_{\mathrm{e}}^{2} \leq A$, avoiding catastrophe and providing a thermal background to chose for the Weibel instability. For $A<0$, the stable case, infrared catastrophe is inhibited by the presence of ions until $m_{\mathrm{e}} / m_{\mathrm{i}}<-A$ and, with $k \rightarrow 0$, for $-2<A$. However, when $A \leq-2$, the nature of the fluctuations changes. In this case magnetic fluctuations belong to the firehose range which excludes the Weibel mode anyway.

The above spectral energy density is a function of wavenumber $k$. It can, in principle, be used in order to determine the wavelength range in which the Weibel mode propagates on the lowest frequency whistler (or Alfvén) branch. This requires calculation of the rms thermal fluctuation amplitude and inserting it into the Alfvén velocity. We do not perform this simple step here.

\section{Conclusions}

The intent of this note, which we believe having achieved, was a critical examination of the role of the Weibel instability. The Weibel instability, though known for half a century, has only recently been celebrated as a new and apparently important non-dynamo generator of magnetic fields in high temperature plasmas. By theory, it would provide relatively long-scale, $0 \lesssim k_{\perp} \lambda_{\mathrm{e}}<1$, quasi-stationary magnetic fields. The linear growth rate, however, maximises in the short-scale range, close to the electron skin depth (or ion skin depth in the ion equivalent of the Weibel mode, if excited, for instance in high speed ultra-relativistic flows).

We have suggested that another restriction limits the wavelength, i.e. the inhibition of electromagnetic modes to propagate in dense non-magnetised plasmas. On the other hand, thermal fluctuations in such plasmas allow for a low level of rms magnetic field amplitudes which serve as background magnetisation. These will always be present, thereby opening a channel for the Weibel mode to propagate on the lowest frequency Alfvén or whistler mode branches.

At these (quasi-stationary) frequencies, thermal fluctuations were believed to undergo an infrared catastrophe. This is not the case. Taking into account the ion contribution and respecting the further condition that thermal fluctuations evolve in the range of stable plasma states, fluctuation theory is valid up to infinitely long wavelengths. The magnetic spectral energy density behaves analytically.

Though the weak rms magnetic fluctuation background indeed solves the problem of Weibel penetration into a plasma, the Weibel magnetic fields are on scales not much longer than the skin depth. Divergence conditions then require that these fields form vortices of neighbouring antiparallel fields, resembling current layers. These may undergo reconnection (Sundkvist et al., 2007), form islands and thus, in addition to quasilinear (Pokhotelov et al., 2010) and nonlinear (Pokhotelov and Amariutei, 2011) limitation, further limit the Weibel amplitude growth in various ways: by structuring the field, plasma jetting, and heating the plasma until depletion of the anisotropic temperature, which is the free energy source of the instability.

Acknowledgements. This research was part of an occasional Visiting Scientist Programme in 2006/2007 at ISSI, Bern.

Topical Editor I. A. Daglis thanks one anonymous referee for her/his help in evaluating this paper.

\section{References}

Achterberg, A. and Wiersma, J.: The Weibel instability in relativistic plasmas. I. Linear theory, II. Nonlinear theory and stabilization mechanism, Astron. Astrophys. 475, 1-18, doi:10.1051/0004-6361:20065365, 2007.

Akhiezer, A. I., Akhiezer, I. A., Sitenko, R. V., and Stepanov, K. N.: Non-Linear Theory and Fluctuations, in: Plasma Electrodynamics, vol. 2, pp. 116-142, Pergamon Press, Oxford, 1975.

Baumjohann, W. and Treumann, R. A.: Basic Space Plasma Physics (Revised Edition), Imperial College Press, London, chpt. 11, 2012.

Baumjohann, W., Nakamura, R., and Treumann, R. A.: Magnetic guide field generation in collisionless current sheets, Ann. Geophys., 28, 789-793, doi:10.5194/angeo-28-789-2010, 2010.

Bykov, A. M. and Treumann, R. A.: Fundamentals of collisionless shocks for astrophysical application: Relativistic shocks, Astroph. Astron. Rev., 19, 42, doi:10.1007/s00159-011-0042-8, 2011.

Christensen, U. R., Balogh, A., Breuer, D., and Glassmeier, K.-H. (Eds.): Planetary Magnetism, Springer Verlag, Heidelberg New York, 2010.

Fried, B. D.: Mechanism for instability of transverse plasma waves, Phys. Fluids, 2, 337, doi:10.1063/1.1705933, 1959.

Hulot, G., Balogh, A., Christensen, U. R., Constable, C., Mandea, M., and Olsen, N. (Eds.): Terrestrial Magnetism, Springer Verlag, Heidelberg New York, 2011.

Jones, C. A., Thompson, M. J., and Tobias, S. M.: The solar dynamo, Space Sci. Rev., 152, 591-616, doi:10.1007/s11214-0099579-5, 2010.

Landau, L. D. and Lifshitz, E. M.: Fluid Mechanics, Course of Theoretical Physics, volume 6, Pergamon Press, Oxford, 1959.

Landau, L. D. and Lifshitz, E. M.: Electrodynamics of Continuous Media, Course of Theoretical Physics, volume 8, Pergamon Press, Oxford, 1960. 
Medvedev, M. V. and Loeb, A.: Generation of magnetic fields in the relativistic shock of Gamma-Ray Burst sources, Astrophys. J., 526, 697-706, doi:10.1086/308038, 1999.

Pokhotelov, O. A. and Amariutei, O. A.: Quasi-linear dynamics of Weibel instability, Ann. Geophys., 29, 1997-2001, doi:10.5194/angeo-29-1997-2011, 2011.

Pokhotelov, O. A., Sagdeev, R. Z., Balikhin, M. A., Fedun, V. N., and Dudnikova, G. I.: Nonlinear Mirror and Weibel modes: peculiarities of quasi-linear dynamics, Ann. Geophys., 28, 21612167, doi:10.5194/angeo-28-2161-2010, 2010.

Retinò, A., Sundkvist, D., Vaivads, A., Mozer, F., André, M., and Owen, C. J.: In situ evidence of magnetic reconnection in turbulent plasma, Nature Phys., 3, 236-238, doi:10.1038/nhys574, 2007.

Ryu, D., Schleicher, D. R. G., Treumann, R. A., Tsagas, C. G., and Widrow, L. M.: Magnetic fields in the large-scale structure of the Universe, Space Sci. Rev., 164, doi:10.1007/s11214-011-9839-z, 2011.

Sitenko, A. G.: Electromagnetic Fluctuations in Plasma, Academic Press, New York, 1967.
Sundkvist, D., Retinò, A., Vaivads, A., and Bale, S. D.: Dissipation in turbulent plasma due to reconnection in thin current sheets, Phys. Rev. Lett., 99, 025004, doi:10.1103/PhysRevLett.99.025004, 2007.

Treumann, R. A., Nakamura, R., and Baumjohann, W.: Collisionless reconnection: mechanism of self-ignition in thin plane homogeneous current sheets, Ann. Geophys., 28, 1935-1943, doi:10.5194/angeo-28-1935-2010, 2010.

Weibel, E. S.: Spontaneously growing transverse waves in a plasma due to an anisotropic velocity distribution, Phys. Rev. Lett., 2, 83-84, doi:10.1103/PhysRevLett.2.83, 1959.

Widrow, L. M., Ryu, D., Schleicher, D. R. G., Subramanian, K., Tsagas, C. G., and Treumann, R. A.: The first magnetic fields, Space Sci. Rev., 164, doi:10.1007/s11214-011-9833-5, 2011.

Yoon, P. H.: Spontaneous thermal magnetic field fluctuations, Phys Plasmas, 14, 064504-064504-4, doi:10.1063/1.2741388, 2007.

Yoon, P. H. and Davidson, R. C.: Exact analytical model of the classical Weibel instability in a relativistic anisotropic plasma, Phys. Rev. A, 35, 2718-2721, doi:10.1103/PhysRevA.35.2718, 1987. 